\title{
Análisis de la influencia del embalse Hidroeléctrico Mazar en el bioclima de la ciudad de Paute.
}

\author{
Analysis of the influence of the Mazar Hydroelectric reservoir on the \\ bioclimate of the city of Paute.
}

Johnny Javier Pacheco Pacheco. ${ }^{1} \&$ Carlos Marcelo Matovelle Bustos. ${ }^{2}$

Recibido: 03-02-2021 / Revisado: 12-02-2021 /Aceptado: 03-03-2021/ Publicado: 05-04-2021

\begin{abstract}
.
DOI: https://doi.org/10.33262/concienciadigital.v4i2.1626

The construction of a hydroelectric reservoir generates an apparent microclimate; manifested in the variation of the temperature of the surrounding areas. In this research, it is proposed to demonstrate the alteration of the temperature of the urban area of the Paute canton located $25 \mathrm{~km}$ from the Mazar hydroelectric reservoir, through the statistical analysis of the annual climatological data obtained from the National Institute of Meteorology and Hydrology of the Ecuador (INAMHI), collected by the meteorological station M0138 located at the study point. To establish the ranges of variation of the climate and issue recommendations for the bioclimatic architectural design of singlefamily homes in the microclimate generated, it was necessary to model retrospective, current and prospective scenarios, for the prospective scenario, climate data was generated with the METEONORM software and compared with 20-year exponential trend curves. With the climatic data analyzed, we generated scenarios for bioclimate studies. Once the bioclimate studies were obtained, a comparative diagnosis was carried out to identify the effects of the microclimate on the hours of comfort, cold and heat. It is observed that the influence of the reservoir generates climate change, directly altering the bioclimate of the city of Paute, reducing the hours of comfort. We conclude that the generated microclimate represents a problem in the bioclimate of the city, for which
\end{abstract}

\footnotetext{
${ }^{1}$ Universidad Católica de Cuenca, Maestría en Construcciones con Mención en la Administración de la Construcción Sustentable, Azuay, Ecuador, johnny.pacheco.37@est.ucacue.edu.ec, https://orcid.org/00000001-7993-7653

${ }^{2}$ Universidad Católica de Cuenca, Grupo de Investigación en Geociencias, Ambiente y Recursos Naturales, Azuay, Ecuador, cmmatovelleb@ucacue.edu.ec,https://orcid.org/0000-0003-2267-0323
} 
measures must be taken to mitigate and adapt to the effects it will have on current and future buildings.

Keywords: Reservoir, biosol, temperature, comfort, microclimate.

\section{Resumen.}

Introducción. La construcción de un embalse hidroeléctrico, genera un aparente microclima; manifestado en la variación de la temperatura de las áreas circundantes. En la presente investigación, se plantea demostrar la alteración de la temperatura de la zona urbana del cantón Paute ubicada a $25 \mathrm{Km}$. del embalse de la hidroeléctrica Mazar, mediante el análisis estadístico de los datos climatológicos anuales obtenidos del Instituto Nacional de Meteorología e Hidrología del Ecuador (INAMHI). Objetivo. Establecer el impacto generado por el embalse de la hidroeléctrica Mazar en el bioclima de Paute en retrospectiva, presente y prospectiva, para poder emitir recomendaciones para el diseño arquitectónico, que permita mitigar y adaptar los edificios. Metodología. El diseño de investigación en retrospectivo fue cuantitativo en base a la estación meteorológica M0138, con estos datos de partida se realiza una investigación cuasi experimental generando curvas de comportamiento y usando un programa de simulación climática (Meteonorm) para la obtención de resultados. Resultados. Para establecer los rangos de variación del clima y emitir recomendaciones del diseño arquitectónico bioclimático de las viviendas unifamiliares en el microclima generado fue necesario modelar escenarios retrospectivos, actuales y prospectivos, para el escenario prospectivo se generó datos del clima con el software Meteonorm y se comparó con curvas de tendencias exponenciales a 20 años. Con los datos climáticos analizados generamos escenarios para estudios de bioclima, una vez obtenidos los estudios del bioclima se realizó un diagnóstico comparativo para identificar los efectos del microclima sobre las horas de confort, frio y calor. Se observa que la influencia del embalse genera un cambio climático, alterando directamente el bioclima de la ciudad de Paute reduciendo las horas de confort. Conclusión. Se concluyó que el microclima generado representa un problema en el bioclima de la ciudad, por lo cual se deberá tomar medidas para la mitigación y adaptación ante los efectos que tendrá en las edificaciones actuales y futuras.

Palabras claves: Embalse, biosol, temperatura, confort, microclima.

\section{Introducción.}

Siendo prioridad de los gobiernos contar con medios de producción de energía limpia y amigable con el entorno, se ha optado por la construcción de centrales hidroeléctricas que están dentro de la producción de energía que menos contaminación causan al medio ambiente de manera directa, aunque es necesario considerar efectos secundarios como las posibles variaciones de temperatura en el microclima de la zona de influencia. 
"Los recursos hídricos son una fuente importante de vida para los seres humanos. Entre sus numerosos beneficios, permiten generar energía eléctrica limpia para un porcentaje importante de la población de América Latina y el Caribe. De hecho, el 50\% de la matriz de generación eléctrica de la región depende de este recurso" (OLADE, 2019).

Debido a ello durante los últimos treinta años se han construido en el país algunos proyectos hidroeléctricos, siendo el proyecto Hidroeléctrico Mazar uno de los más importantes en la región, el cuál utiliza el caudal del Río Paute, está ubicado al sur-este del Ecuador sobre los límites de las Provincias de Azuay y Cañar, perteneciente al cantón Sevilla de Oro de la primera provincia, como se puede observar en la fig. 1. El sitio de las obras se encuentra en el km 105 de la vía Cuenca-Paute-Guarumales-Méndez, entre las cotas altitudinales de 2008 a $2400 \mathrm{msnm}$. Administrativamente el proyecto está bajo la jurisdicción de las provincias de Azuay y Cañar, en los cantones Azogues, Paute, Guachapala, El Pan y Sevilla de Oro; iniciando trabajos en mayo del 2005, actualmente se tiene en funcionamiento la Presa Mazar, y se realiza generación de energía en las turbinas. El funcionamiento de la central aporta con 160 MW para el Sistema Nacional Interconectado.

"Se ha ahondado en la influencia de los proyectos en la vida de las comunidades campesinas e indígenas -en especial-, y desde el campo ecologista se ha trabajado el cómo las grandes obras se constituyen en factores de cambio del sistema ambiental de las zonas de influencia" (Lucero, 2016)

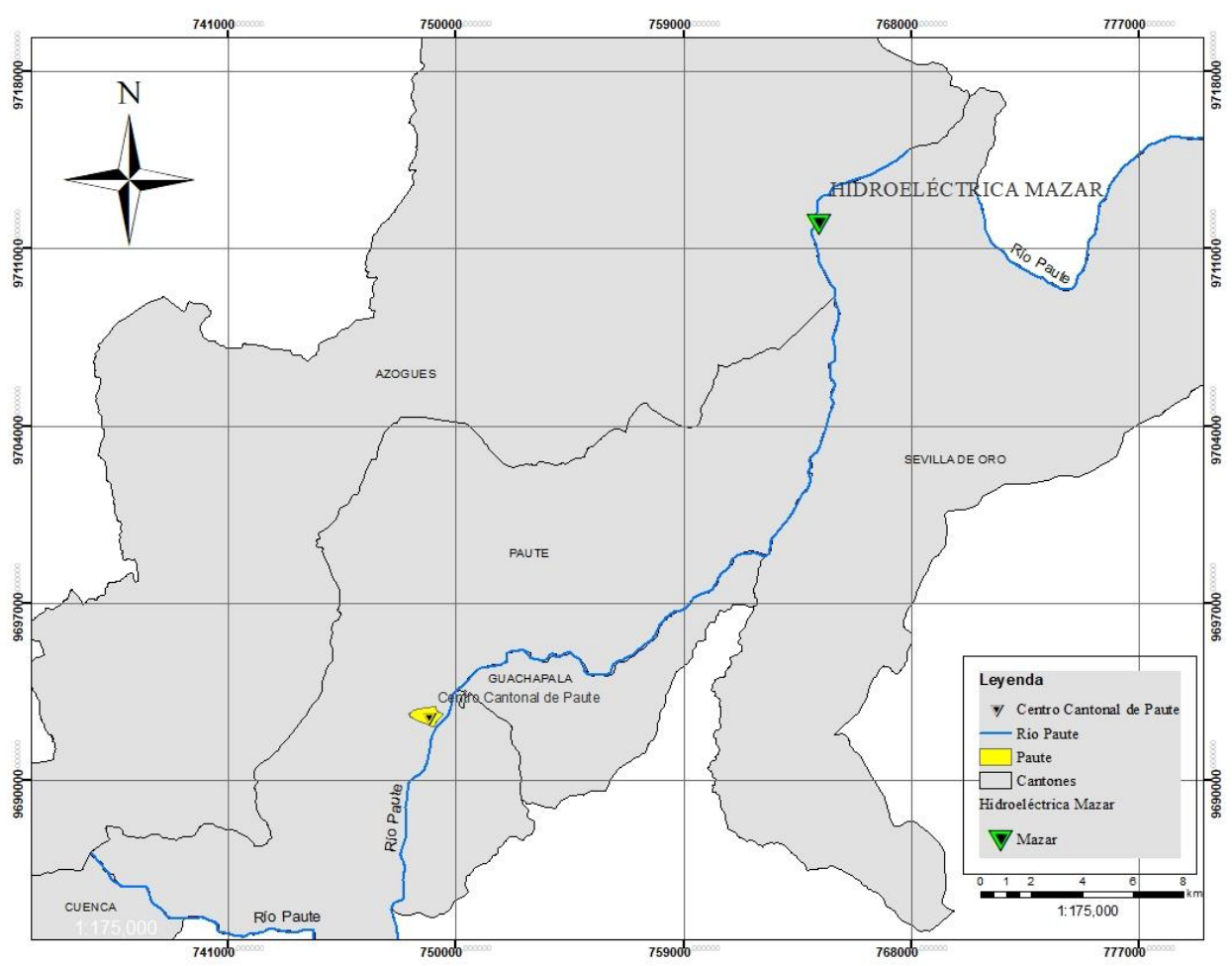

Figura 1. Ubicación de la Hidroeléctrica Mazar y el centro cantonal de Paute.

Fuente: Elaboración propia. 
El proyecto hidroeléctrico Mazar entra en funcionamiento en el año 2010, creándose en esta fecha un lago artificial en el Río Paute, "la creación de lagos artificiales conlleva la aparición de notables alteraciones en los microclimas locales cuyo origen debe relacionarse ante todo con el incremento de la humedad atmosférica en su entorno inmediato" (GARCIA CODRON, 1994). Por tanto, existe un área afectada en el cual se crea un microclima debido al cambio de temperaturas. En el presente estudio se demuestra las variaciones de temperatura antes del embalse (<2010) y luego del embalse $(>2010)$ analizando datos históricos del clima obtenidos mediante anuarios meteorológicos de 1982 a 2013 de la institución INAMHI específicamente de la estación M0138 denominada Paute, ubicada en las siguientes coordenadas; Latitud: $2^{\circ} 48^{\prime} 00,00^{\prime \prime} \mathrm{S}$, Longitud: 78 45'46,00" W y altitud: $2194 \mathrm{msnm}$. donde se encuentra un total de 5014 habitantes en la zona urbana del centro cantonal de Paute según el último censo del 2010, siendo el asentamiento con mayor población dentro del área de acción del microclima, por tanto, la zona con mayor construcción de viviendas unifamiliares.

"Las pequeñas modificaciones climáticas a escala local inducidas por el hombre han tenido lugar a través de cambios en la composición atmosférica y en las condiciones de la superficie terrestre. Alguno de estos impactos parece ser el resultado o al menos tener como causa última la transformación de considerables extensiones de suelo forestal, de pastos y de cultivos en medios acuáticos artificiales de agua dulce, es decir, en embalses." (Astorga, 1994)

El estudio de los factores del clima, es de gran importancia en la actualidad y más aún las proyecciones de los mismos al futuro, simulando la ocurrencia de eventos que influyen directamente con el ser humano. El estudio de estos escenarios es fundamental, pues los diseñadores podrían dar soluciones de confort desde la etapa de anteproyecto de una edificación frente a fenómenos como el calentamiento global y variación de temperatura.

"El cambio climático es una realidad, actualmente se vive una situación autodestructiva, principalmente debido a la mano del hombre que ha contaminado el planeta, causando efectos dañinos para su propia salud y el bien de la naturaleza. La Organización de las Naciones Unidas (ONU) ha publicado que el aumento de la temperatura en el planeta, el derretimiento de los polos, el aumento del nivel del mar, etc. Es principalmente debido a la acción humana por la emisión de gases contaminantes hacia la atmosfera; principalmente el dióxido de carbono; CO2" (Grupo Intergubernamental de Expertos sobre el Cambio Climático, 2013).

"Para lograr una mejor comprensión de las interacciones entre el sistema climático terrestre, los ecosistemas y las actividades humanas, la comunidad científica ha desarrollado y utilizado diversas herramientas y metodologías. Una de ellas es el uso de "escenarios", los cuales pueden definirse como descripciones coherentes, internamente consistentes y convincentes de un posible estado futuro del mundo" (Eduardo et al., 2016).

El confort se refiere de manera más puntual a un estado de percepción ambiental momentáneo casi instantáneo, el cuál ciertamente está determinado por el estado de salud 
del individuo, pero además por muchos otros factores, los cuales se pueden dividir en forma genérica en dos grupos: Los factores endógenos, internos o intrínsecos del individuo, y factores exógenos o externos y que no dependen del individuo; entre los cuales podemos destacar la temperatura ambiente.

Para las proyecciones se utilizó el software Meteonorm, "este software usa modelos estocásticos para valores que tienen las mismas propiedades estadísticas, para calcular los valores diarios utiliza modelos de Aguiar y Collares Pereira y las cadenas de Markov basándose en la suposición de la distribución de frecuencias de radiación solar. Para generar valores horarios de temperatura se parte de la "suposición de que la amplitud de las variaciones diurnas de temperatura es aproximadamente proporcional a la amplitud de la radiación solar diaria y, por lo tanto, el perfil de temperaturas se obtiene transformando el perfil de radiación solar". Meteonorm maneja series históricas y se puede acceder a escenarios que brinden información futura acerca del clima de la zona de estudio". (Morillon Gálvez et al., 2013)

Meteonorm es una base de datos que contiene información climatológica de aproximadamente 1000 estaciones meteorológicas alrededor del planeta, es una herramienta que permite a los usuarios acceder a bases de datos interpolados en prospectiva. Este software usa modelos estocásticos para valores que tienen las mismas propiedades estadísticas, para calcular los valores diarios utiliza modelos de Aguiar y Collares Pereira y las cadenas de Markov basándose en la suposición de la distribución de frecuencias de radiación solar. Se compara las proyecciones realizadas por el programa Meteonorm con las curvas de comportamiento exponenciales obtenidas por el análisis de datos generados por la estación meteorológica M0138.

"La tendencia al aumento de la temperatura en la Tierra y la alteración de las variables climáticas son los resultados inequívocos del próximo cambio climático" (McCarthy, J., et al, 2001). Se obtuvieron las predicciones de temperatura en el programa de simulación antes mencionado a un Representative Concentration Pathways 8.5 (RCP 8.5) el cual nos da un escenario pesimista-realista del comportamiento de temperaturas medias mensuales. El RCP 8.5 combina supuestos sobre una alta población y un crecimiento de los ingresos relativamente lento con tasas modestas de cambio tecnológico y mejoras en la intensidad energética, lo que lleva a largo plazo a una alta demanda de energía y emisiones de GEI. RCP8.5 corresponde por tanto a la vía con las mayores emisiones de gases de efecto invernadero (Riahi, et al, 2011).

"Es importante considerar los modelos a una escala regional que permitan aplicar análisis de variaciones globales en determinadas áreas específicas con mejor resolución" (Jiménez, et al, 2020).

"El análisis comparativo presentado, permite identificar el panorama bioclimático que se tiene en las costas mexicanas, para poder emitir recomendaciones para el diseño arquitectónico, que permita mitigar y adaptar los edificios." (Wolfskill, Valenzuela, 2017). 
Al realizar este análisis, se demostró el cambio de temperaturas en la zona de influencia del embalse y por lo tanto en el centro cantonal de Paute, donde se realizan la mayor parte de actividades constructivas. Con las curvas de tendencia y las simulaciones generadas se obtuvo información de las variantes de temperatura que se utiliza en el método del biosol, el cual es un software para el estudio del bioclima, control solar e iluminación natural, los datos de entrada requeridos son los promedios mensuales de temperaturas máximas y mínimas, latitud, longitud y altura de la localidad: obteniendo como resultado el diagrama de isorrequerimientos de condiciones de frio, calor o confort.

"Con los años, los embalses de la zona boreal y templada, tienden a presentar emisiones netas de gases con efecto invernadero, muy equiparables a las de lagos de similares características y si se comparan los balances del embalse con el ecosistema terrestre al que reemplazaron, no siempre el balance es peor" (Palau y Alonso, 2008).

Con el presente estudio se establecen los datos de partida para que se generen las recomendaciones de diseño bioclimático que permitan mitigar o aprovechar la variación de la temperatura producto de la intervención del hombre en la naturaleza, en este caso particular del embalse para proyecto hidroeléctrico, lo que nos permite aportar a la generación de proyectos de viviendas sustentables.

\section{Metodología.}

Según Sierra Bravo (1992), la presente investigación tiene los siguientes enfoques;

\section{TIPOS Y NIVELES DE INVESTIGACIÓN}

\begin{tabular}{|c|c|c|}
\hline $\begin{array}{l}\text { Según su } \\
\text { finalidad }\end{array}$ & Aplicada & $\begin{array}{l}\text { Los resultados permiten resolver problemas de cambio } \\
\text { climático aplicado en la construcción bioclimática. }\end{array}$ \\
\hline \multirow{2}{*}{$\begin{array}{l}\text { Según su } \\
\text { alcance } \\
\text { temporal }\end{array}$} & Retrospectiva & Se analizan datos de temperaturas desde 1982 al 2013. \\
\hline & Prospectiva & $\begin{array}{l}\text { Se analizan datos de temperatura del presente y se proyecta } \\
\text { temperaturas a } 20 \text { años. }\end{array}$ \\
\hline $\begin{array}{l}\text { Según su } \\
\text { profundidad }\end{array}$ & Explicativa & $\begin{array}{l}\text { La investigación permite estudiar el impacto de un embalse } \\
\text { hidroeléctrico en el Bioclima de una ciudad. }\end{array}$ \\
\hline $\begin{array}{l}\text { Según las } \\
\text { fuentes }\end{array}$ & Secundarias & $\begin{array}{l}\text { La investigación se realizó a partir de datos ya existentes, } \\
\text { recogidos por el INAMHI por medio de una estación } \\
\text { meteorológica y publicado en sus anuarios. }\end{array}$ \\
\hline $\begin{array}{l}\text { Según el } \\
\text { carácter }\end{array}$ & Cuantitativas & $\begin{array}{l}\text { Procesamos datos de temperatura obtenidos de una estación } \\
\text { meteorológica. }\end{array}$ \\
\hline $\begin{array}{l}\text { Según su } \\
\text { naturaleza }\end{array}$ & Cuasiexperimentales & $\begin{array}{l}\text { Proyectamos tendencias o comportamientos de temperatura } \\
\text { por medio de software o curvas de tendencia, para emitir } \\
\text { comparaciones. }\end{array}$ \\
\hline
\end{tabular}

Tabla 1. Tipos y niveles de investigación.

Fuente: Elaboración propia. 
Las variables de análisis son de carácter cuantitativo que permiten establecer rangos de variaciones estadísticas, cuyos datos fueron recolectados aplicando el muestreo aleatorio por conglomerados de tipo probabilístico.

El análisis de tendencias de una serie temporal de datos da resultados muy interesantes en cuanto a la variación de su comportamiento en un lapso de tiempo determinado, como se puede observar en el estudio realizado por Alemu, Z.A., \& Dioha, M.O (2020) en el que vincula el cambio climático con las tendencias de temperatura en una zona específica. En el estudio propuesto se realizó un análisis retrospectivo de las temperaturas máximas y mínimas medias obtenidas de la estación meteorológica M0138 de los anuarios meteorológicos del INAMHI desde 1982 al 2013 que existen publicados oficialmente. El objetivo del análisis pretende demostrar el cambio de temperatura e inclinación de las curvas de tendencia tanto de las temperaturas medias máximas y de las temperaturas medias mínimas. Con los datos climáticos obtenidos se aplicaron en el software Biosol, el cual permite analizar los parámetros de confort higrotérmico diariamente en el sector de estudio, lo que posibilita la utilización de energía solar como fuente para el consumo energético dentro de una vivienda.

Para obtener el escenario prospectivo del clima en el sector de estudio se utilizó el programa Meteonorm, dicho programa usa métodos de interpolación espacial proporciona datos de los factores climáticos como son: humedad, radiación, temperatura y permite realizar proyecciones a futuro. Con el objetivo de comparar y validar la información obtenida se propone la utilización de las curvas de tendencia de comportamiento para obtener temperaturas prospectivas para el año 2040, la línea de tendencia de mayor confiabilidad que usamos es la exponencial ya que su valor $\mathrm{R}$ cuadrado es el más cercano a 1 , siendo la más fiable. Con estos datos podemos realizar un análisis bioclimático a 20 años para comparar escenarios en las horas de frio, calor o confort por la influencia del embalse de la hidroeléctrica Mazar. El procedimiento para análisis del confort que se empleó para esta investigación fue el propuesto por (Preciado y Morillon, 2010) el mismo que se basa en un análisis bioclimático partiendo de la premisa de eficiencias luminosas en superficies verticales y horizontales mismos que generan la gráfica solar y también los días de frio, calor o confort que se presenten en el año de estudio.

\section{Resultados.}

El embalse de la hidroeléctrica fue llenado y puesto en funcionamiento a lo largo del año 2010, por lo que se realiza un análisis de las temperaturas antes del embalse desde enero de 1982 hasta diciembre de 2009 de los anuarios meteorológicos publicados año a año por el Instituto Nacional de Meteorología e Hidrología del Ecuador, obteniendo curvas de comportamiento tanto para la temperatura media máxima y la temperatura media mínima. 


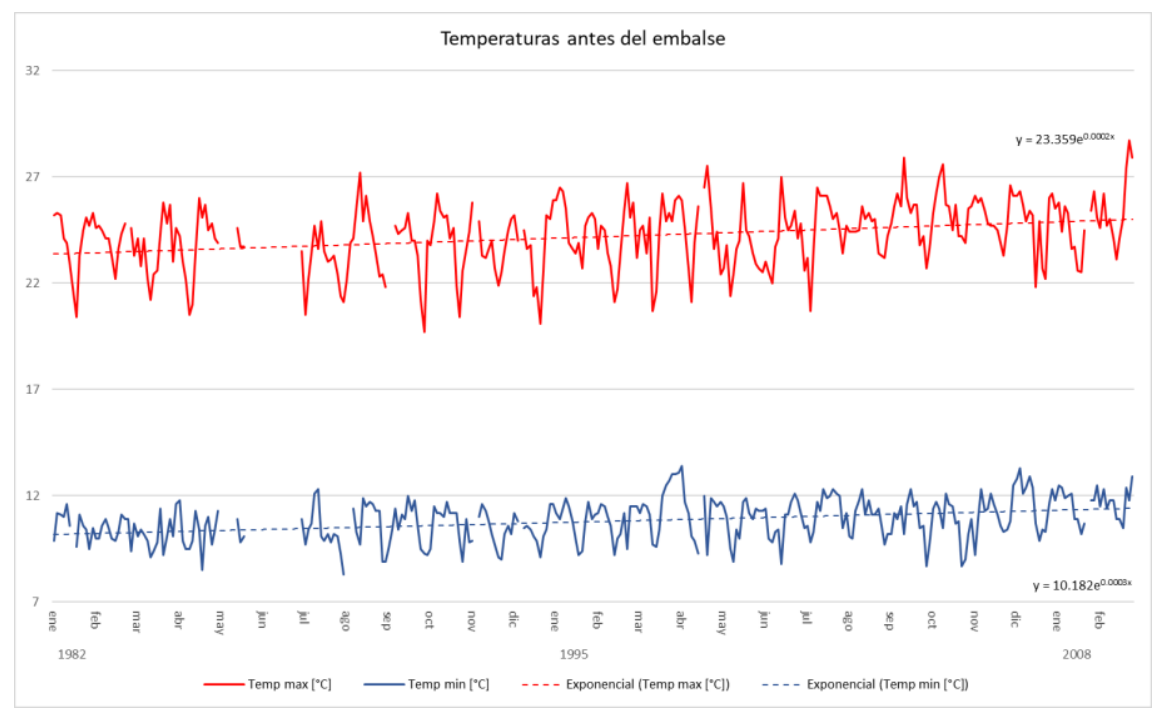

Figura 2. Temperaturas medias máximas y mínimas (1982-2009).

Fuente: Elaboración propia.

Del análisis de las temperaturas medias tanto máximas como mínimas se obtuvo una curva de comportamiento exponencial siendo las fórmulas las siguientes.

$$
y=23.359 * e^{0.0002 * x}
$$

Donde "x" es el valor de la interacción del tiempo, "y" es la temperatura media máxima.

$$
y=10.182 * e^{0.0003 * x}
$$

Donde " $\mathrm{x}$ " es el valor de la interacción del tiempo, "y" es la temperatura media mínima.

Se realizó un análisis desde enero de 2011 a diciembre de 2013 que fue la última emisión de los anuarios meteorológicos por parte del Instituto Nacional de Meteorología e Hidrología del Ecuador obteniendo los siguientes resultados.

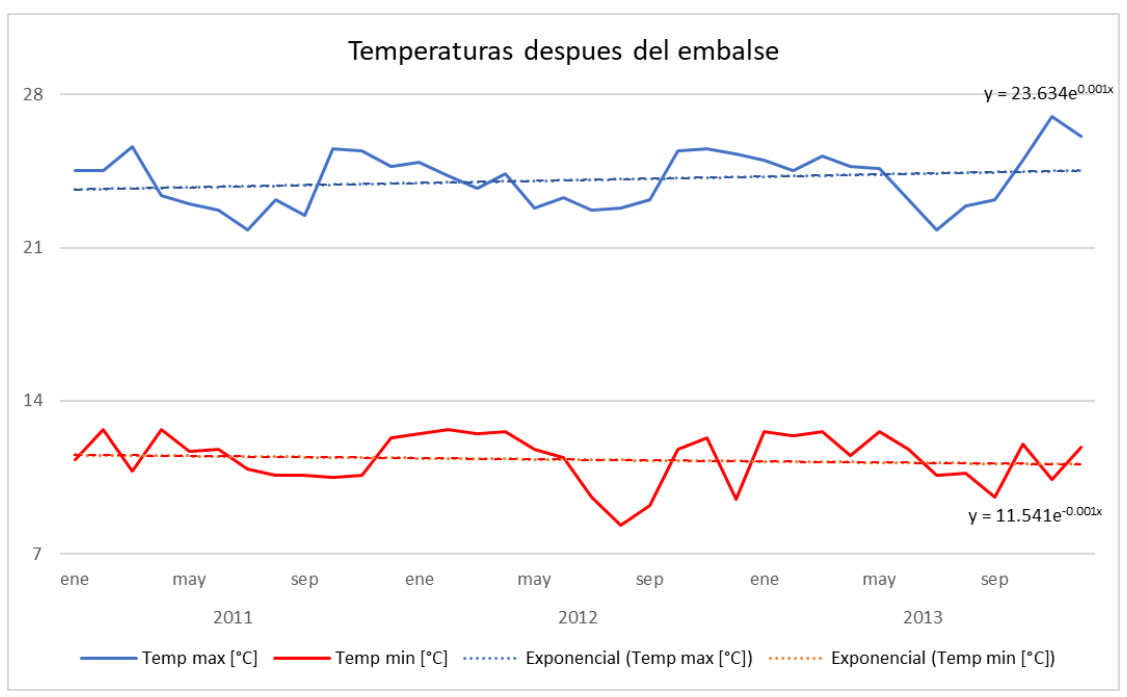

Figura 3. Temperaturas medias máximas y mínimas (2011-2013).

Fuente: Elaboración propia. 
Del análisis de las temperaturas medias tanto máximas como mínimas se obtuvo una curva de comportamiento exponencial siendo las fórmulas las siguientes.

$$
y=23.634 * e^{0.001 * x}
$$

Donde " $x$ " es el valor de la interacción del tiempo, "y" es la temperatura media máxima.

$$
y=11.541 * e^{-0.01 * x}
$$

Donde " $\mathrm{x}$ " es el valor de la interacción del tiempo, "y" es la temperatura media mínima.

Por medio de las ecuaciones (1), (2), (3) y (4) obtenemos las curvas de tendencia exponencial de temperaturas medias máximas y mínimas, que nos permite realizar una comparación grafica de las mismas en un periodo de 5 años luego de la realización del embalse y comparamos resultados entre lo proyectado y lo real.

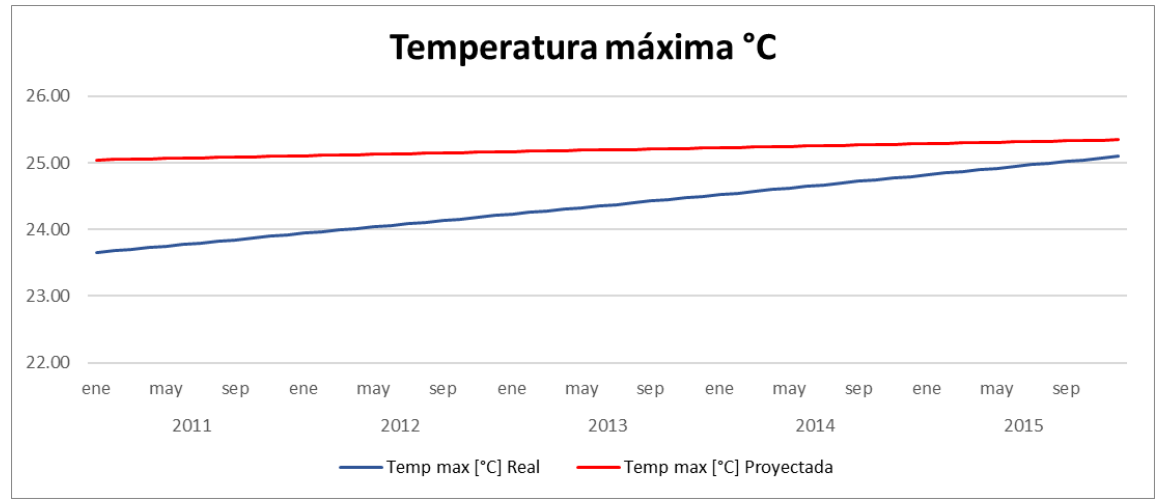

Figura 4. Temperaturas medias máximas proyectadas y reales entre los años 2011-2015.

Fuente: Elaboración propia.

De acuerdo a la gráfica de tendencias de temperaturas podemos observar una variación en enero de 2011 de $-1.39^{\circ} \mathrm{C}$ entre la temperatura media máxima proyectada y la real afirmando que existió un enfriamiento de la zona debido al lago artificial o embalse de la hidroeléctrica, el ángulo de crecimiento de la curva se modificó notablemente tendiendo a temperaturas más altas en futuro.

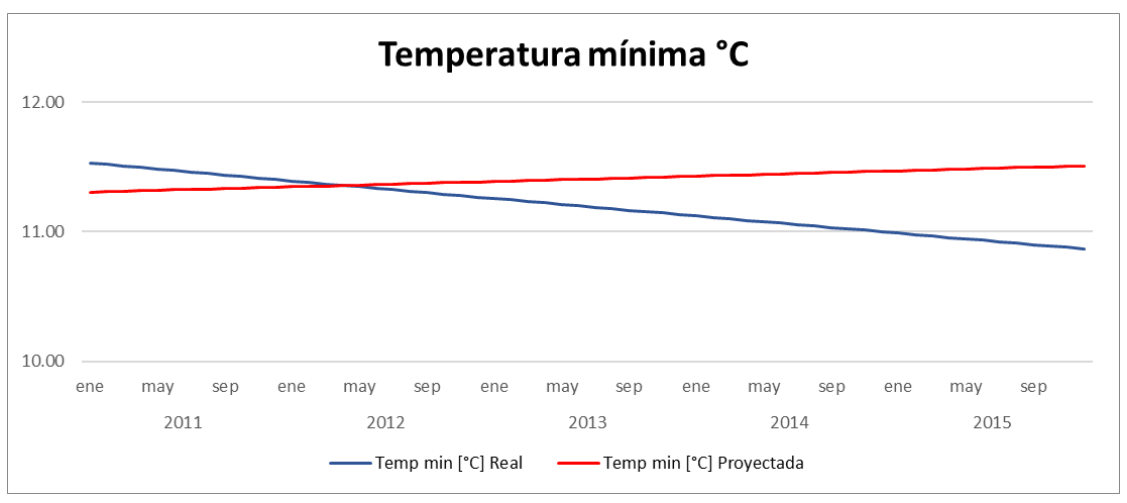

Figura 5. Temperaturas medias mínimas proyectadas y reales entre los años 2011-2015.

Fuente: Elaboración propia. 
De acuerdo a la gráfica de tendencias de temperaturas podemos observar una variación en enero de 2011 de $0.22^{\circ} \mathrm{C}$ entre la temperatura media mínima proyectada y la real afirmando que existió una desviación del ángulo de crecimiento de la curva tendiendo hacer más frio en la zona.

Los resultados obtenidos de la simulación del programa Meteonorm son los de la siguiente tabla.

\begin{tabular}{ccc}
\hline \multicolumn{3}{c}{ PROYECCIÓN DE TEMPERATURAS AÑO 2040} \\
MES & Temp $\max \left[{ }^{\circ} \mathrm{C}\right]$ & Temp $\min \left[{ }^{\circ} \mathrm{C}\right]$ \\
\hline ene & 24.00 & 8.20 \\
$\mathrm{feb}$ & 24.40 & 9.30 \\
$\mathrm{mar}$ & 23.20 & 9.30 \\
$\mathrm{abr}$ & 22.40 & 8.80 \\
may & 23.00 & 9.20 \\
jun & 22.30 & 9.60 \\
jul & 23.10 & 8.20 \\
ago & 22.40 & 7.00 \\
sep & 22.30 & 7.40 \\
oct & 24.30 & 9.00 \\
nov & 24.40 & 8.00 \\
dic & 24.30 & 8.00 \\
\hline
\end{tabular}

Tabla 2. Temperaturas máximas y mínimas, medias del año 2040.

Fuente: Elaboración propia.

Con los resultados obtenidos en perspectiva (1982-2009), presente (2011-2020) y prospectiva 2040 mediante el programa Meteonorm y mediante una proyección según la curva de comportamiento se pueden realizar las siguientes comparaciones.

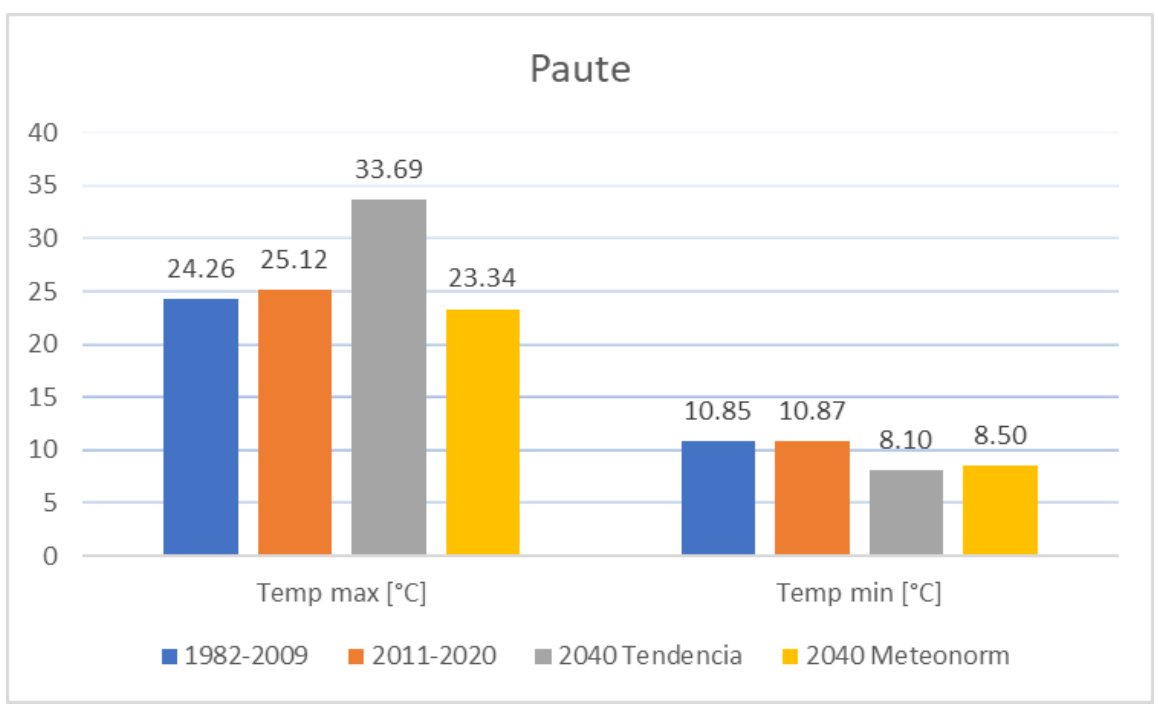

Figura 6. Tabla de evidencia del cambio de temperaturas en la ciudad de Paute.

Fuente: Elaboración propia. 


\section{EDigital}

ISSN: 2600-5859

WwW.concienciadigital.org

Vol. 4, N², p. 39-54, abril-junio, 2021

En las figuras 7 y 8 podemos observar los escenarios retrospectivo y presente, que las horas predominantes son las de confort durante la media mañana y tarde, y las horas de frio durante la noche y madrugada.

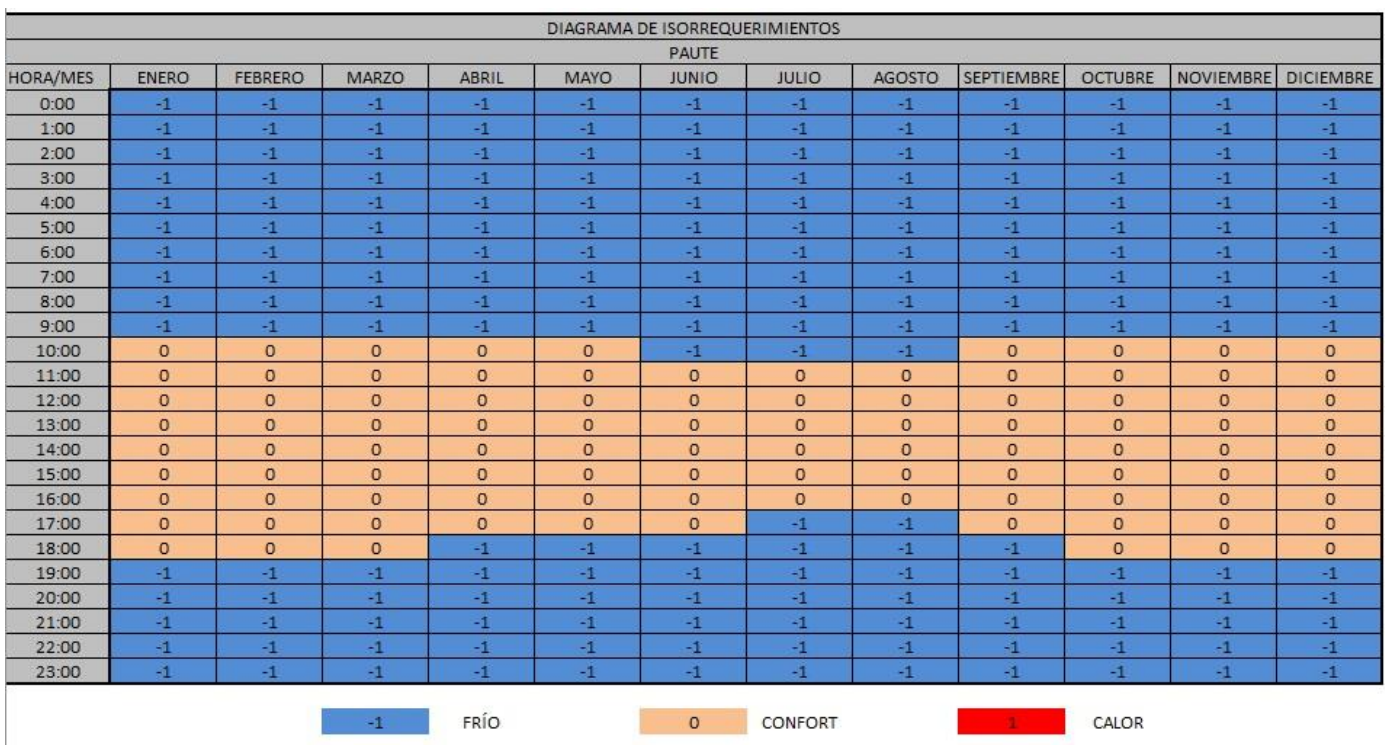

Figura 7. Diagrama de isorrequerimientos del Bioclima retrospectivo 1982-2009 en la ciudad de Paute.

Fuente: Elaboración propia.

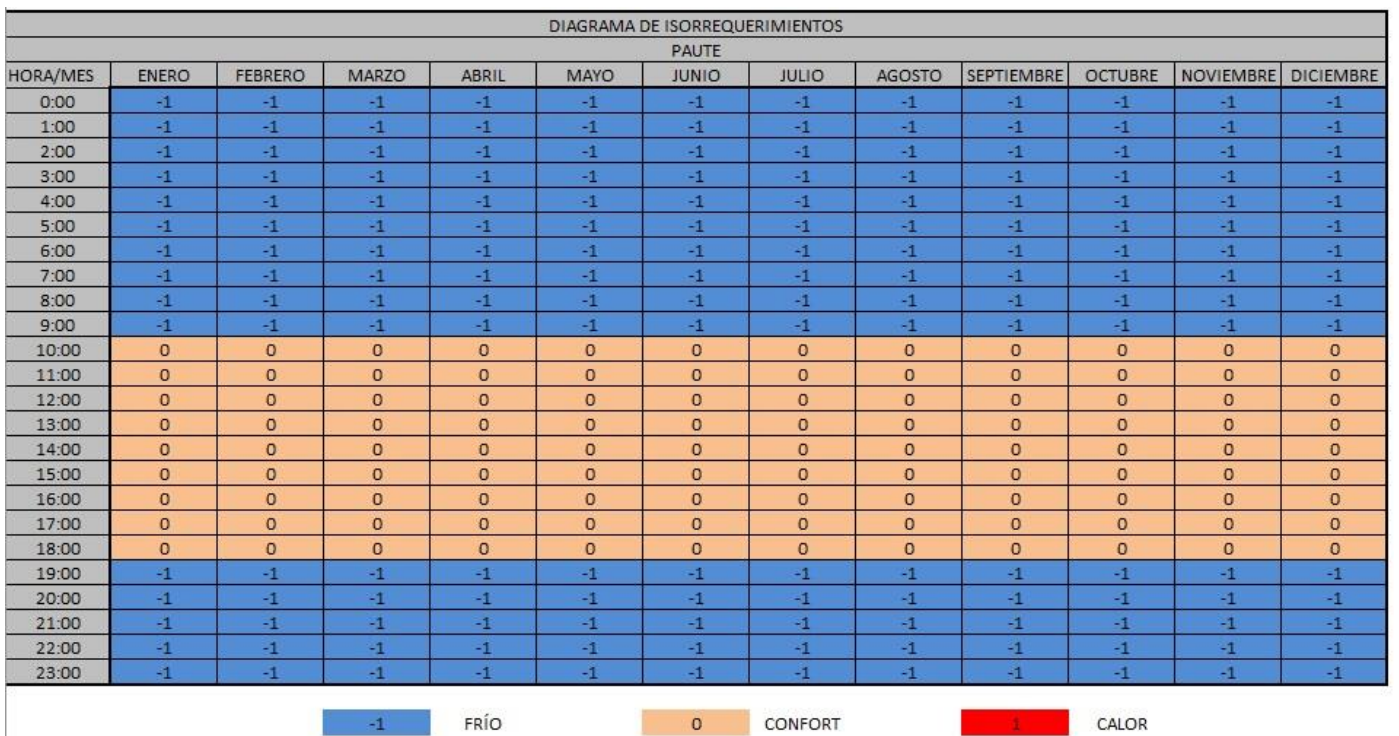

Figura 8. Diagrama de isorrequerimientos del Bioclima presente (2011-2020) en la ciudad de Paute.

Fuente: Elaboración propia.

La ciudad de Paute tiene un incremento marcado en su temperatura máxima y un descenso menos notorio en su temperatura mínima, pero sus horas de confort suelen ser más constantes tanto en el bioclima retrospectivo, presente y escenario prospectivo obtenido del programa Meteonorm (fig. 9). 
ISSN: 2600-5859

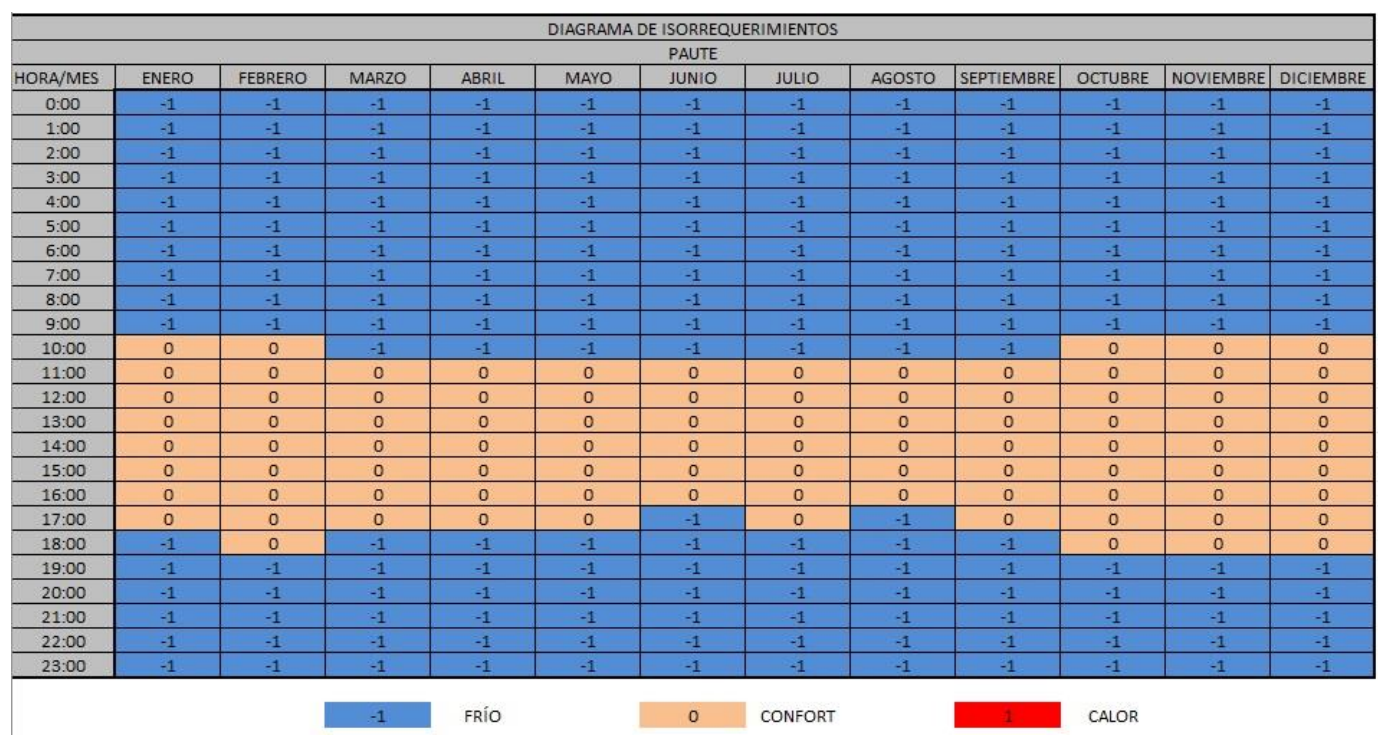

Figura 9. Diagrama de isorrequerimientos del Bioclima prospectivo 2040 METEONORM en la ciudad de Paute.

Fuente: Elaboración propia.

En el escenario prospectivo obtenido mediante curvas de comportamiento (fig. 10) las horas de calor remplazan a las horas de confort de $11 \mathrm{~h} 00$ a 16h00. El escenario prospectivo al 2040 se pierden horas de confort siendo cambiadas por horas de calor las cuales en los escenarios anteriores no se habían presentado hasta la fecha, causada por el incremento de temperatura por la irradiación del sol de la laguna artificial.

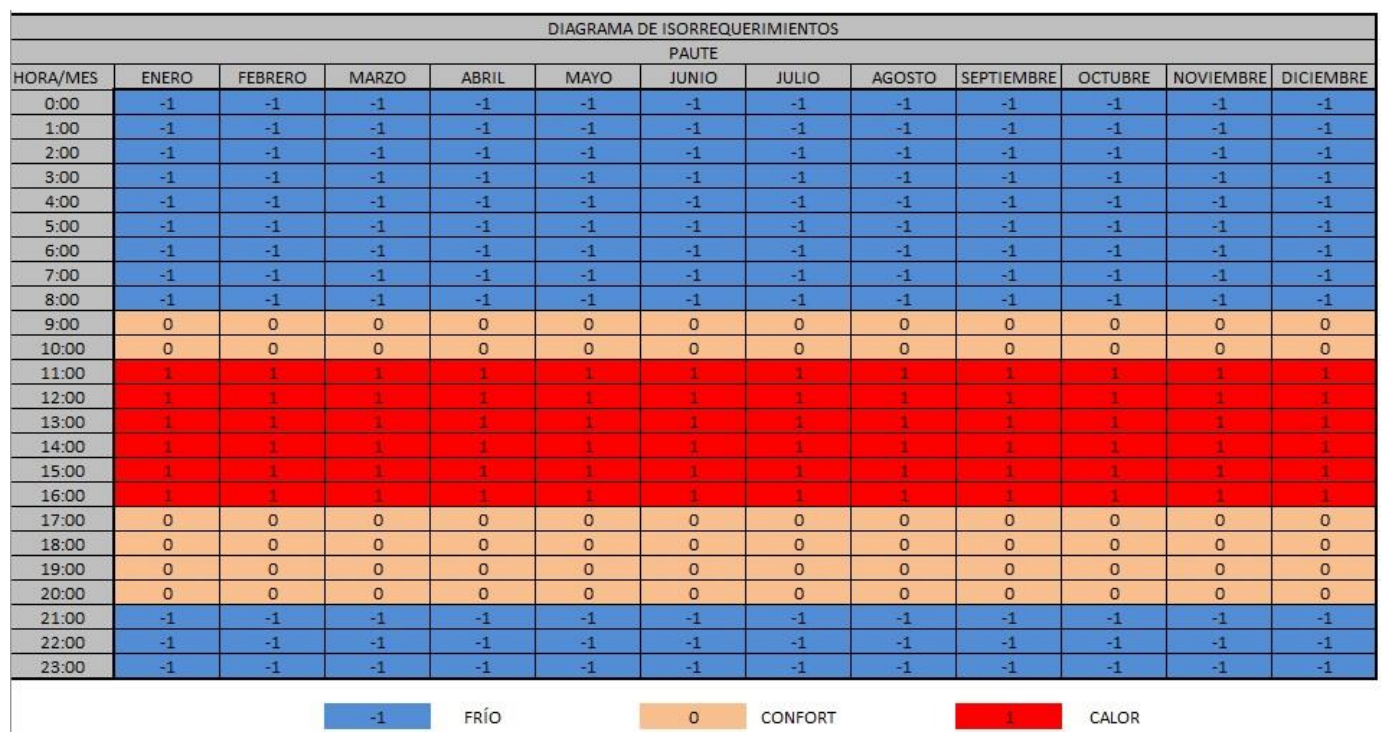

Figura 10. Diagrama de isorrequerimientos del Bioclima del año 2040 proyectado por curvas de tendencia en la ciudad de Paute.

Fuente: Elaboración propia.

En la siguiente figura se muestran los resultados anteriores sintetizados en forma de grafico para poder comparar e identificar el impacto del cambio climático en el 
microclima generado por el represamiento de agua que sufre el bioclima en la ciudad de Paute.

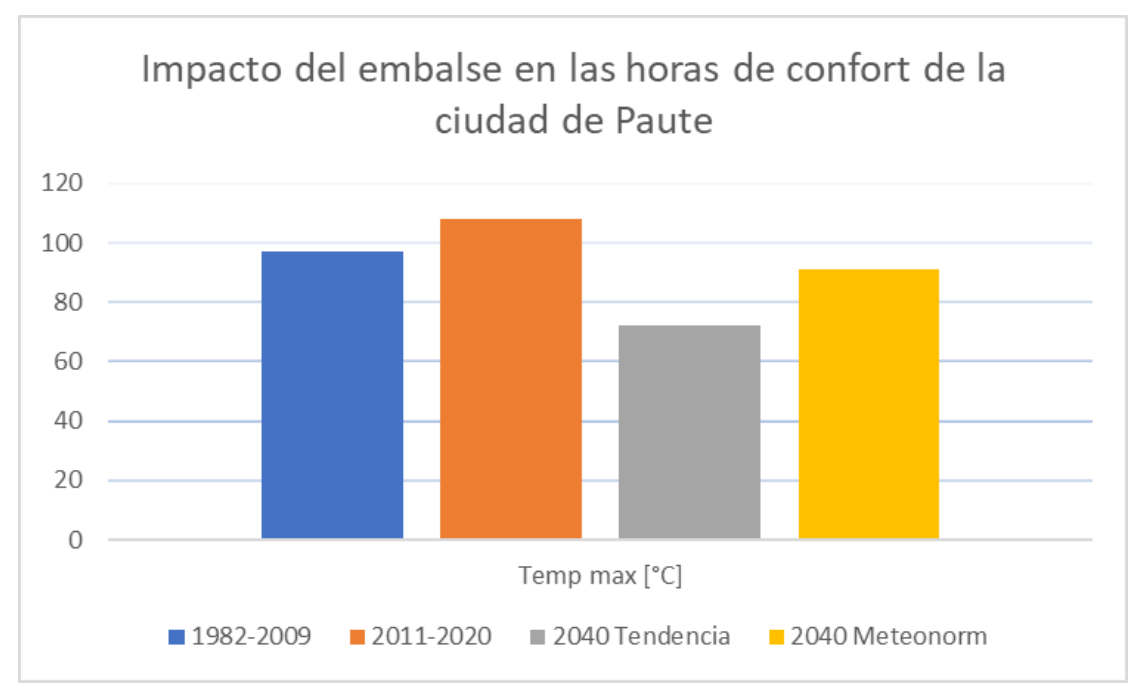

Figura 11. Gráfico comparativo del impacto del embalse hidroeléctrico en las horas de confort, medido en horas.

Fuente: Elaboración propia.

En la figura 11 se puede observar que en las horas de confort aumentan en el escenario presente (2011-2020) debido al incremento de temperaturas medias máximas teniendo en promedio 9 horas diarias de confort de $10 \mathrm{~h} 00$ a 18h00, en el escenario prospectivo (2040) de tendencia de curvas de comportamiento de temperaturas las horas de confort disminuyen notablemente en comparación con el resto de escenarios analizados.

\section{Conclusiones.}

- Con el análisis de las tendencias de temperatura y la comparación entre la data antes de la implementación del embalse y posterior al funcionamiento del mismo se valida los impactos en el microclima que puede generar el embalse de la hidroeléctrica Mazar en la ciudad de Paute, en prospectiva aumentan las temperaturas máximas por lo que aumentara las horas de disconfort durante el día. En el escenario presente se tiene una reducción de temperaturas inmediatamente luego de ser llenado el embalse, sin embargo, la curva de comportamiento de temperaturas máximas se vuelve más pronunciada generando el aumento de temperaturas al pasar de los años.

- Al contar con una proyección bioclimática de la zona, se pueden proponer distintos sistemas de climatización pasivas y estrategias que al tomarse en cuenta pueden ayudar a brindar una sensación de confort en condiciones adversas a este, según el diagrama de isorrequerimientos.

- Al presentarse horas de calor durante el día, los diseñadores arquitectónicos deben plantearse nuevas estrategias en sus diseños ya que en el escenario retrospectivo y presente no tenían que lidiar con horas de calor.

- Se debe proponer un reordenamiento territorial en las que se implementen normas 
de construcción basadas en confort higrotérmico por bioclima para adaptar mejor a la ciudad en los años venideros.

AGRADECIMIENTOS: El presente artículo es parte del trabajo de investigación y titulación del Programa de Maestría en Construcción con Mención en Administración de la Construcción Sustentable de la Universidad Católica de Cuenca, por ello agradezco a todos y cada uno de los instructores por los conocimientos e información brindados para la elaboración del trabajo.

\section{Referencias bibliográficas.}

Alemu, Z.A., Dioha, M.O. Climate change and trend analysis of temperature: the case of Addis Ababa, Ethiopia. Environ Syst Res 9, 27 (2020). https://doi.org/10.1186/s40068-020-00190-5

Astorga González, A.F. (1994), Posibles cambios climáticos debidos a los embalses construidos en las cabeceras de los ríos de montaña, serie geográfica vol. 4, Pp. 45-54.

Eduardo, G., Porras, A., \& Jácome, P. S., (2016), Proyecciones climáticas de precipitación y temperatura para ecuador, bajo distintos escenarios de cambio climático.

García Codrón, J.C. (2014), El impacto climático de los embalses cantábricos, en serie geográfica vol. 4. Pp. 33-42.

Grupo Intergubernamental de Expertos sobre el Cambio Climático, 2013. Cambio Climático 2013 Bases Físicas, s.l.: Naciones Unidas, s.f. Naciones Unidas. [En línea] Available at: http://www.un.org/es/sections/issues-depth/climatechange/index.html

INAMHI (1982-2013), Anuarios meteorológicos de 1982 a 2013, Quito, Ecuador.

Jimenez S., Aviles A., Galán L., Flores A., Matovelle C., Vintimilla C. (2020) Support Vector Regression to Downscaling Climate Big Data: An Application for Precipitation and Temperature Future Projection Assessment. In: Fosenca C E., Rodríguez Morales G., Orellana Cordero M., Botto-Tobar M., Crespo Martínez E., Patiño León A. (eds) Information and Communication Technologies of Ecuador (TIC.EC). TICEC 2019. Advances in Intelligent Systems and Computing, vol 1099. Springer, Cham. https://doi.org/10.1007/978-3-030-357405_13

Lucero, F., (2016): Reconfiguración de paisaje y grandes proyectos. el caso del proyecto hidroeléctrico mazar, Azuay-Ecuador. 
McCarthy, J., et al.: IPCC Climate Change: Impacts, Adaptation and Vulnerability. Contribution of Working Group II to the Third Assessment Report of the Intergovernmental Panel on Climate Change (2001).

Morillon, D., Gálvez, D., Preciado, O., \& (Ed), R. A., (2013): Ingeniería de la energía solar para la sustentabilidad (p. 249).

OLADE, (2019), Panorama energético de América Latina y el Caribe 2019, Quito, Ecuador.

Palau, A. y M. Alonso, (2008), Embalses y cambio climático, Monografías de Endesa, Dirección de Medio Ambiente y Desarrollo Sostenible, Endesa, Lleida, pp. 47.

Preciado y Morillón, (2010): Biosol: Software para el estudio del Bioclima, Control solar e iluminación natural. IV Conferencia Latino Americana de energía Solar (IVISES_CLA) U XVIII Simposio Peruano de Energía Solar (XVII-SPES) 5 noviembre, Cusco, Perú.

Riahi, K., Rao, S., Krey, V. et al. RCP 8.5-A scenario of comparatively high greenhouse gas emissions. Climatic Change 109, 33 (2011). https://doi.org/10.1007/s10584011-0149-y

Wolfskill, L., Valenzuela, K. (2017): Impacto del cambio climático en el Bioclima de las costas mexicanas: retrospectiva, presente y prospectiva. XLI Semana Nacional de Energía Solar 2017. Pp.13-18, ANES, ISES, AMIE. Del 6 al 8 de octubre de 2017, Guadalajara, Jalisco, México.

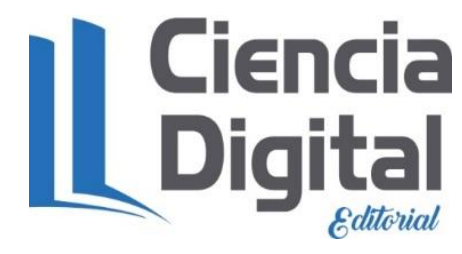




\section{PARA CITAR EL ARTÍCULO INDEXADO.}

Pacheco Pacheco, J. J., \& Matovelle Bustos, C. M. (2021). Análisis de la influencia del embalse Hidroeléctrico Mazar en el bioclima de la ciudad de Paute. ConcienciaDigital, 4(2), 39-54. https://doi.org/10.33262/concienciadigital.v4i2.1626

\section{Liencia}

El artículo que se publica es de exclusiva responsabilidad de los autores y no necesariamente reflejan el pensamiento de la Revista Conciencia Digital.

El artículo queda en propiedad de la revista y, por tanto, su publicación parcial y/o total en otro medio tiene que ser autorizado por el director de la Revista Conciencia Digital.

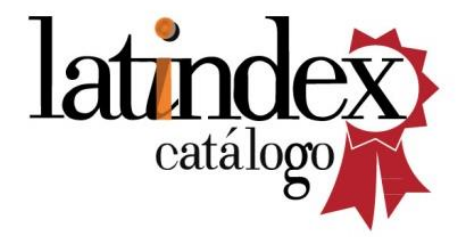

